\title{
A Electric Vehicle Powertrain Simulation and Test of Driving Cycle Based on AC Electric Dynamometer Test Bench
}

\author{
YANG Sheng-bing \\ College of Automotive Engineering \\ Wuhan University of Technology \\ Technology Center, Dongfeng Motor \\ Corporation \\ Wuhan, China \\ 149184028@qq.com \\ ZHANG Guo-guang \\ Technology Center, Dongfeng Motor \\ Corporation \\ Wuhan, China
}

\author{
LI Zhen-zhen \\ College of Automotive Engineering \\ Wuhan University of Technology \\ Hubei Key Laboratory of Advanced \\ Technology of Automotive Parts \\ Wuhan, China \\ 450648031@qq.com \\ DENG Yuan-fa \\ Technology Center, Dongfeng Motor \\ Corporation \\ Wuhan, China
}

\author{
XU Feng \\ College of Automotive Engineering \\ Wuhan University of Technology \\ Hubei Key Laboratory of Advanced \\ Technology of Automotive Parts \\ Wuhan, China
}

\author{
WANG Jun \\ College of Automotive Engineering of \\ Jilin University \\ Jilin, China
}

\begin{abstract}
The AC( Alternating Current) electric dynamometer test bench consists of an AC electric dynamometer, a traction motor, a frequency converter ACS800,a motor controller, a power analyzer WT3000 and etc. By simulation of ADVISOR, relationship between traction motor speed $n$ and load torque $\mathrm{Tz}$ in ECE-EUDC driving cycle was obtained, which was synchronously transmitted to the motor controller and the dynamometer converter by the CAN interface card. Experiment shows the real driving cycle state can be simulated on the AC electric dynamometer test bench. Result of energy consumption economy by ADVISOR and test bench is basically consistent, which is of great significance on EV(Electric Vehicle) development research.
\end{abstract}

Keywords-electric vehicle; AC electric dynamometer; driving cycle; simulation; test

\section{INTRODUCTION}

AC( Alternating Current) electric dynamometer is a new type of mechanical and electrical integrated high-performance dynamometer. It uses three phase squirrel-cage asynchronous motor as the loading equipment. ABB frequency converter provides variable frequency drive power for asynchronous motor, and accurately controls its torque and speed. AC electric dynamometer has a good capability of power-driven and power absorption, cooperating with automatic measurement and control system, it can carry out complex dynamic test and simulation test ${ }^{[1,2]}$.

Energy consumption per kilometer of EV(Electric Vehicle) in specific driving cycle is one of the important performance indexes of EV. To test energy consumption per kilometer of $\mathrm{EV}$ in a vehicle, special experiment equipment is needed. It has rigorous requirements for experiment site, vehicles and instrument ${ }^{[7]}$. Therefore, AC electric dynamometer test bench

The authors acknowledge Hubei Key Laboratory of Advanced Technology of Automotive Parts and the Fundamental Research Funds for the Central Universities for their financial support (Grant NO. 2011-IV-078) and Dongfeng Electric Vehicle Co. LTD. for their equipment support. is applied to conduct driving cycle simulation and test, which is more effective and cheaper.

This article established a kind of system based on AC dynamometer test bench for vehicle driving cycle simulation and test. The relationship between traction motor speed $n$ and load torque $T_{z}$ in ECE-EUDC driving cycle was obtained by time step utilizing advisor. They were synchronously transmitted to the related parts by the CAN interface card, which realized driving cycle simulation and test.

This paper is divided into five major sections as follows. Section one opens with the structure of AC electric dynamometer test system. The system design including principle of resistance simulation and driving cycle simulation on ADVISOR is introduced in section two. Section three lists energy consumption test and its calculation principles. Section four related to vehicle driving cycle test in test bench and the results of simulation and test. The last section draws a conclusion.

\section{AC ELECTRIC DYNAMOMETER TEST SYSTEM}

Shown in Figure 1, it's block diagram of AC electric dynamometer test system, which consists of the following equipment: Industrial PC, Siemens PLC,AC electric dynamometer, frequency converter ACS800,programmable DC power module DCS800,WT3000 power analyzer and etc.

AC electric dynamometer test system controls ABB converter and power by Siemens PLC; Programmable DC power supply power for dynamometer and speed and torque were given by ACS800;In the stage of data acquisition,WT3000 power analyzer was applied to record motor torque, speed, efficiency and other related parameters to provide data for subsequent analysis. This system can be used 
in the vehicle simulation and test of driving cycle to meet requirements of the experiment.

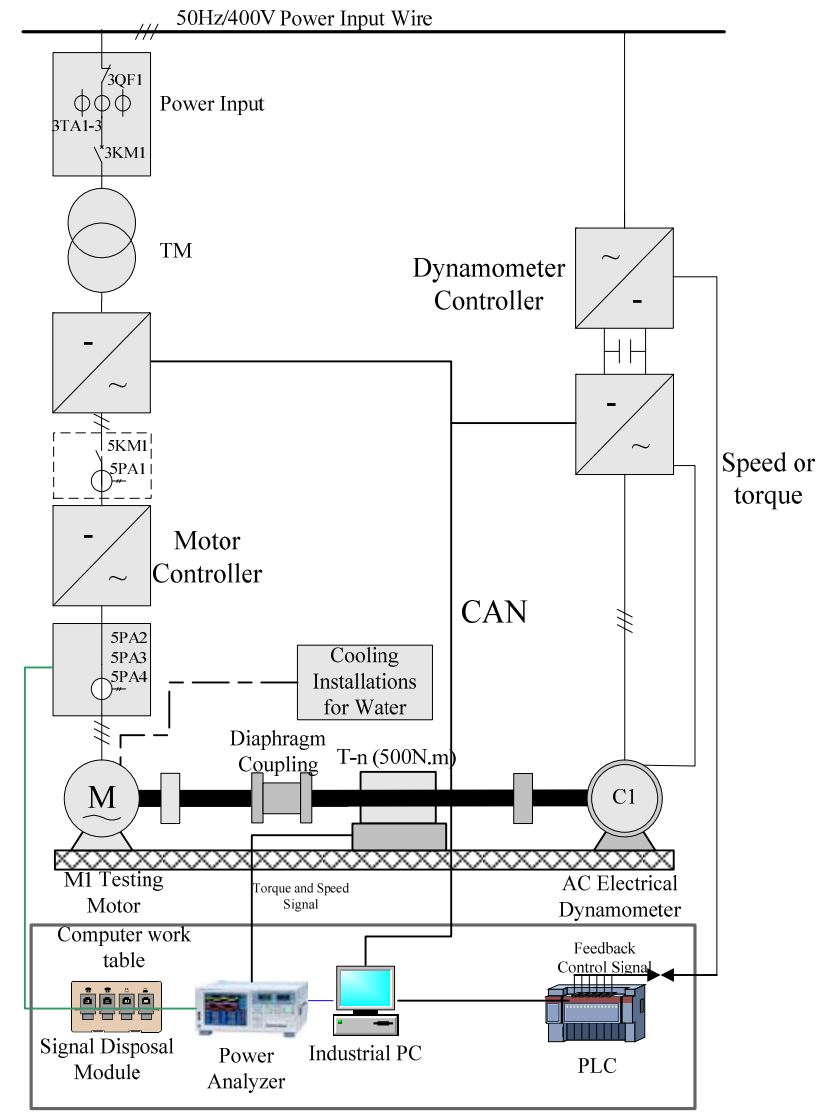

Figure 1. Block diagram of AC Electric Dynamometer Test System

\section{SimUlATION OF DRIVING RESISTANCE}

A. Principle of Resistance Simulation

When a vehicle driving in the road, the resistance is ${ }^{[4]}$

$T_{z}=\frac{r}{i_{g} i_{0} \eta}\left(m g f+\frac{C_{D} A}{21.5} u_{a}^{2}+m g i+\delta m \frac{d u}{d t}\right)$

$F_{f}$ is rolling resistance, $F_{w}$ is air friction, $F_{i}$ is gradient resistance, $F_{j}$ is acceleration resistance, $\mathrm{m}$ is vehicle mass, $f$ is rolling resistance coefficient, $C_{D}$ is air resistance coefficient, $A$ is vehicle windward area, $u_{a}$ is vehicle running speed, $i$ is ramp angle, $\delta$ is vehicle rotating mass reduction coefficient.

Force on the wheel that traction motor transferred to is

$$
F_{z}=\sum F=\frac{T_{z} i_{g} i_{0} \eta}{r}
$$

$T_{z}$ is traction motor load torque, $i_{g}$ is gear box transmission ratio, $i_{0}$ is final ratio, $\eta$ is transmission efficiency, $r$ is wheel radius. By formula (1) and (2), traction motor load torque is shown as formula (3).

$$
T_{z}=\frac{r}{i_{g} i_{0} \eta}\left(m g f+\frac{C_{D} A}{21.5} u_{a}^{2}+m g i+\delta m \frac{d u}{d t}\right)
$$

While the relation between traction motor speed $\mathrm{n}$ and vehicle running speed $u_{a}$ is

$$
n=2.653 \frac{u_{a} i_{g} i_{0}}{r}
$$

The meaning of $i_{g}, i_{0}$ and $\mathrm{r}$ are showed in formula (2).

\section{B. Driving cycle simulation by ADVISOR}

ADVISOR is one kind of MATLAB/SIMULINK based simulation software, which provides graphical user interface (GUI) for user to modify vehicle parameters. Users are capable of modifying parameters through its source file (.m file in MATLAB) or parameter dialog box, which is terrifically convenient to define different vehicle models.

On the foundation of actual vehicle tracking test, every country has formulated some typical driving cycle to simulate the car running status and evaluates its energy consumption economy in corresponding driving cycle by its hundredkilometer power consumption ${ }^{[3]}$.Some European countries uses UDDS driving cycle, while ECE-EUDC driving cycle is typically used in China.

TABLE I. MAIN PARAMETER OF EV

\begin{tabular}{|c|c|c|c|c|c|c|}
\hline $\begin{array}{c}\text { vehicle mass } \\
(\mathrm{kg})\end{array}$ & $\begin{array}{c}\text { windward } \\
\text { area A } \\
(\mathrm{m} 2)\end{array}$ & $\begin{array}{c}\text { wheel radius } \mathrm{r} \\
(\mathrm{m})\end{array}$ & $\begin{array}{c}\text { rolling resistance } \\
\text { coefficient }\end{array}$ & $\begin{array}{c}\text { air resistance } \\
\text { coefficient }\end{array}$ & $\begin{array}{c}\text { vehicle rotating mass } \\
\text { reduction coefficient }\end{array}$ & $\begin{array}{c}\text { transmission } \\
\text { efficiency }\end{array}$ \\
\hline 1144 & 2 & 0.282 & 0.009 & 0.335 & 1.04 \\
\hline
\end{tabular}

Main parameters of EV this paper used is shown as table 1.These EV Parameters were configured by modifying the corresponding $\mathrm{m}$ file. In ECE-EUDC driving cycle, appropriate time step of simulation was needed. Considering simulation precision and LabVIEW program synchronization issues, $100 \mathrm{~ms}$ time step was chosen ${ }^{[6]}$.By simulation and 
formula (3) and (4), the relationship between traction motor speed $\mathrm{n}$ and load torque $T_{z}$ is obtained, which is shown as Figure 2.
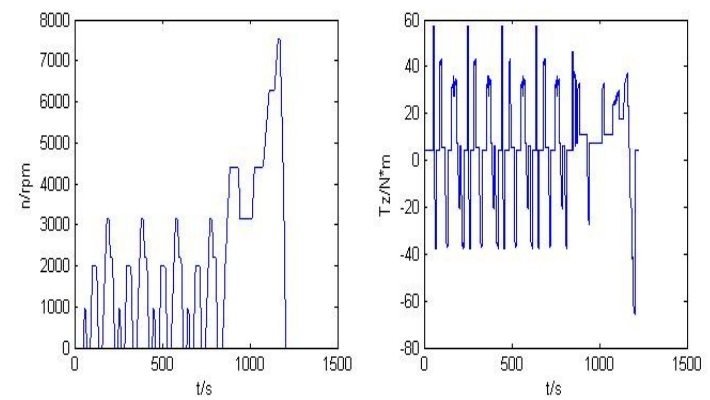

Figure 2. Relationship between traction motor speed $\mathrm{n}$ and load torque $\mathrm{Tz}$

\section{ENERGY CONSUMPTION TEST}

\section{A. Energy consumption test}

Vehicle evaluation index of energy consumption economy is on the basis of certain driving cycle, which is measured by energy consumption in certain vehicle driving mileage. Pure electric vehicles take battery as the only energy source. In related references, driving range, capacity consumption per kilometer, energy consumption per kilometer, driving range per capacity and etc are used as vehicle evaluation index of energy consumption economy, among which energy consumption per kilometer is much more direct and accurate $^{[7]}$.It means battery power consumption per kilometer.

\section{B. calculation principles of energy consumption}

According to Automobile Theory, vehicle power balance formula is ${ }^{[4,7,8]}$

$$
T_{z}=\frac{r}{i_{g} i_{0} \eta}\left(m g f+\frac{C_{D} A}{21.5} u_{a}^{2}+m g i+\delta m \frac{d u}{d t}\right)
$$

According to the above definition about energy consumption per kilometer of pure electric vehicle, therefore, energy consumption per kilometer is shown as formula (6)

$$
E p=\frac{\int_{0}^{t} P(t)}{s}
$$

$E_{p}$ is energy consumption per kilometer, $\mathrm{t}$ is driving time, $S$ is distance of certain driving cycle. By formula (6), electric vehicle energy consumption per kilometer can be calculated by ADVISOR simulation results.

When testing in the test bench, the product of battery terminal voltage and discharge current each time step is integrated, energy consumption of EV is obtained as formula (7) shows

$$
E=\int_{0}^{t} U(t) I(t) d t
$$

Energy consumption per kilometer by test bench is showed as formula (8).

$$
E_{t}=\frac{W}{s}
$$

By these formulas, we can test energy consumption economy in the test bench.

\section{VEHICLE DRIVING CYCLE TEST}

When conducting driving cycle test in test bench, traction motor speed $\mathrm{n}$ was transmitted to motor controller and load torque $T_{z}$ to dynamometer converter ACS800 synchronously according to relationship between traction motor speed $n$ and load torque $T_{z}$ which can be acquired in ECE-EUDC driving cycle by the result of the simulation in ADVISOR. This test has no meaning if speed and torque command were not sent synchronously ${ }^{[5]}$.As a result, synchronization was the key to simulation and test. Utilizing virtual instrument technology LabVIEW and its multithreaded programming environment, synchronization can be realized.

The testing system used CAN interface card PCI-9820I With two CAN ports. According to realize the time synchronization, CAN protocol will have speed command input to the traction motor controller, and the same time the load torque of traction motor will be sent to dynamometer motor frequency conversion controller ACS800 through CANOPEN protocol. With its two CAN ports, combined with LabVIEW message queue and state machine, speed and torque command can be synchronously sent. The method of combining the state machine and message queue could avoid the phenomenon of deadlock produced due to competition resources. Through the debugging system, the synchronous sending speed and torque instruction is realized. Shown as Figure 3, it's the simulation and test flow chart of vehicle driving cycle. 


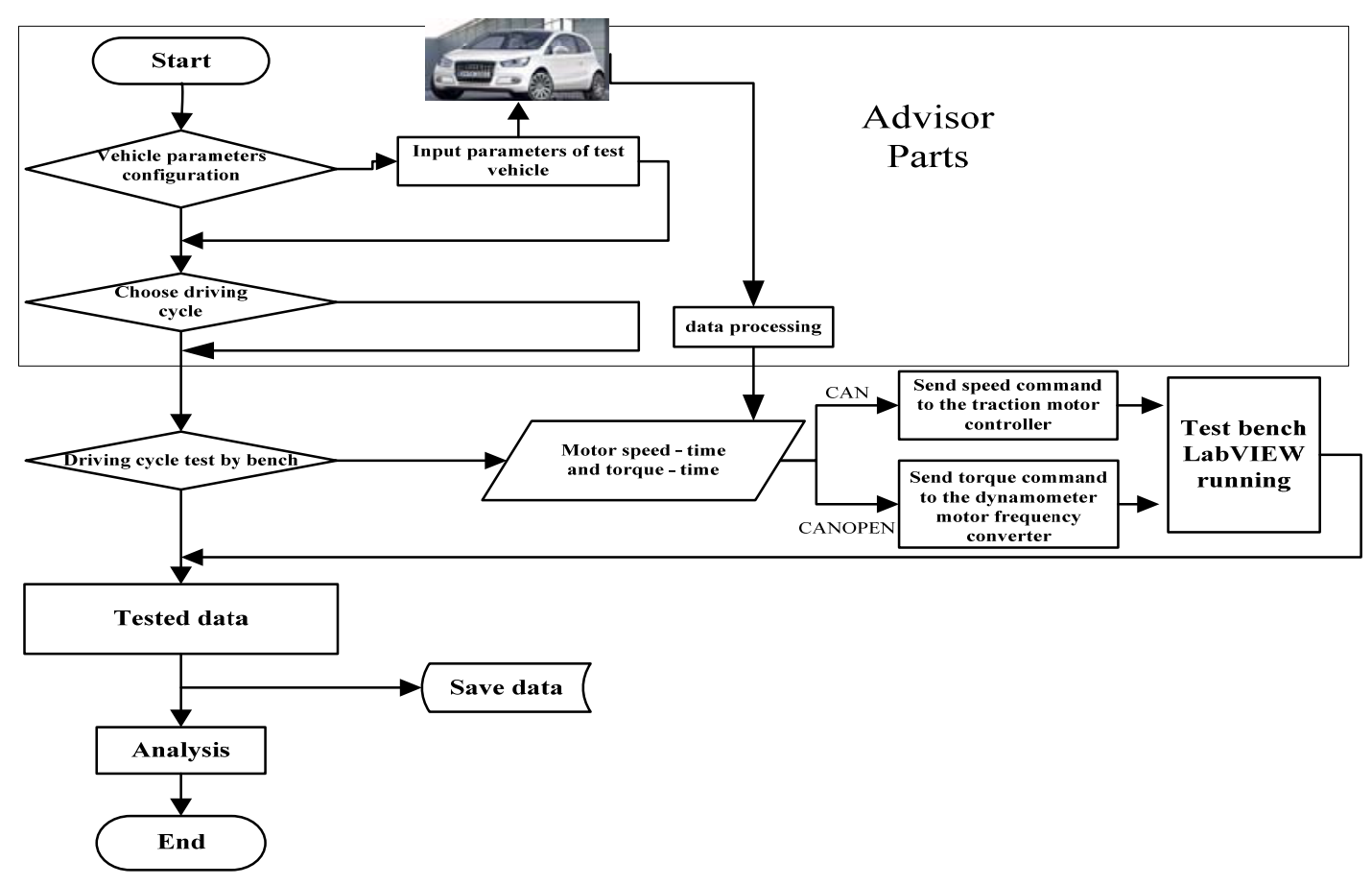

Figure 3. Simulation and test flow chart of vehicle driving cycle

According to the result of test bench simulation and test, energy consumption per kilometer was calculated, which was $0.33 \mathrm{~kW} \cdot \mathrm{h} / \mathrm{km}$.By advisor simulation, it's $0.28 \mathrm{~kW} \cdot \mathrm{h} / \mathrm{km}$.The two results are basically consistent. Tests show that the system run steady and reliable, which has a high application prospect. The results of the study have guiding significance in the development of EV and the matching optimization design of its powertrain at a certain extent.

\section{CONCLUSION}

The paper applied graphical programming language LabVIEW to develop PC software, by ADVISOR, relationship between traction motor speed $n$ and load torque $T_{z}$ in ECEEUDC driving cycle was obtained and synchronously sent from LabVIEW front panel downwards for the reason of LabVIEW's supporting for multithreading technology. Test results in test bench were basically consistent with simulation results. The research is of great significance for the development of electric vehicle.

\section{REFERENCES}

[1] Jesus Arellano-Padilla,Greg M.Asher,etc.Control of an AC Dynamometer for Dynamic Emulation of Mechanical Loads With Stiff and Flexible Shafts[J].IEEE TRANSACTIONS ON INDUSTRIAL ELECTRONICS,VOL.53,NO.4,AUGUST 2006.

[2] ZhuMaotao,WuXin,etc.Development of Measurement and Control System for Vehicular Powertrain Testbench in HEV Applications[C].2011 International Conference on Remote Sensing, Environment and Transportation Engineering, RSETE 2011 Proceedings,p 3063-3067.

[3] Nguyen Phi Hung,Hoang Emmanuel,Gabsi Mohamed.Performance synthesis of permanent-magnet synchronous machines during the driving cycle of a hybrid electric vehicle[J]. IEEE Transactions on Vehicular Technology, v60,n5,p 1991-1998,June 2011.

[4] YU Zhi.Automobile Theory[M]..Beijing : Mechanical Industry Press,2009.(in Chinese)

[5] Poon, Jason J.and etc.Hardware-in-the-loop testing for electric vehicle drive applications[J].IEEE Applied Power Electronics Conference and Exposition- APEC, p 2576-2582,2012.

[6] Jiang Facha,Li Lin,Wang Molin.Simulation and analysis of hybrid electric vehicle powertrain[J].Advanced Materials Research,v 230-232,p 964-967,2011,Frontiers of Manufacturing Science and Measuring Technology.

[7] WANG Zhen-po,YAO Li-min,SUN Feng-chun.Preliminary Study on Evaluation System of EV Energy Consumption Economy[J].Journal of Beijing Institute of Technology,2005,25(6).(in Chinese)

[8] WU Qing-long,ZONG Zhi-jian,LIU Zhong-tu.Simulation and Test of Electric Vehicle Driving Cycle Based on Test Bench[J]. Small \& Special Electrical Machines,2011.(in Chinese) 